Acta Cryst. (1987). A43, 6-13

\title{
Isomorphous Replacement: Effects of Errors on the Phase Probability Distribution
}

\author{
By Thomas C. Terwilliger* and David Eisenberg \\ Molecular Biology Institute and Department of Chemistry and Biochemistry, University of California, \\ Los Angeles, California 90024, USA
}

(Received 7 December 1985; accepted 16 April 1986)

\begin{abstract}
A simple framework for the analysis of the effects of errors in the method of isomorphous replacement is developed. This framework is used to derive phase probability distributions similar to those given by Blow \& Crick [Acta Cryst. (1959). 12, 794-802]. The present analysis suggests an improved means of calculating the mean-square 'lack-of-closure' residuals and shows that they differ by a factor of two for centric and acentric reflections. It is also shown that the lack-of-closure residuals have a straightforward interpretation and that they may be used to estimate the error in the heavy-atom model and the degree of isomorphism between native and derivative structures if anomalous differences have been measured.
\end{abstract}

\section{Introduction}

In the method of isomorphous replacement, information from several sources is generally combined by multiplying the various probability distributions for each phase. Consequently the accuracy of these probability distributions is of some importance and a detailed understanding of the effects of errors on these distributions would be valuable.

An expression often used to calculate a phase probability distribution $P(\varphi)$ for a reflection is (Blow \& Crick, 1959)

$$
P(\varphi) \propto \exp \left[-\left(F_{P H}^{o}-F_{P H}^{c}\right)^{2} / 2 E^{2}\right]
$$

where $\varphi$ is the native phase and $F_{P H}^{o}$ is the observed derivative structure-factor amplitude. $F_{P H}^{c}$ is a calculated derivative structure-factor amplitude, given by

$$
F_{P H}^{c}=\left|F_{P}^{o} \exp (i \varphi)+\mathbf{f}_{\mathbf{H}}\right| \text {. }
$$

Here $F_{P}^{o}$ is the observed native structure-factor amplitude and $\mathbf{f}_{\mathbf{H}}$ is an estimate of the structure factor of the heavy atoms present in the derivative structure but not in the native structure, calculated from a model.

Although it is clear that the choice of $E^{2}$ in $(1 a)$ is crucial, the proper value of $E^{2}$ is not obvious. In practice (Ten Eyck \& Arnone, 1976), the value of $E^{2}$

* Present address: Department of Biochemistry and Molecular Biology, University of Chicago, 920 East 58th Street, Chicago, Illinois 60637, USA.

0108-7673/87/010006-08\$01.50 generally used is the mean-square value of the lackof-closure' residual, $\left\langle\left(F_{P H}^{o}-F_{P H}^{c}\right)^{2}\right\rangle$, where $F_{P H}^{c}$ is the derivative structure-factor amplitude calculated using $(1 b)$ at the 'best' native phase.

In this paper, we develop a simple framework for the analysis of the various errors in the isomorphous replacement method. We show that phase probability distributions similar to those given by Blow \& Crick (1959) may be derived, beginning with very basic assumptions. This derivation shows that current methods of estimating $E^{2}$ are justified except that the value of $E^{2}$ for centric reflections is twice that for acentric reflections. We also suggest a somewhat improved method of estimating $E^{2}$. Finally, we show how the lack-of-closure residuals may be used to estimate the lack of isomorphism between native and derivative structures as well as the error in the heavyatom model if anomalous differences have been measured.

\section{Errors in calculated and measured derivative structure-factor amplitudes}

Even if the native phase $\varphi$ is known, the calculated derivative structure-factor amplitude $F_{P H}^{c}$ for a particular reflection calculated using $(1 b)$ is not generally equal to the measured derivative structure-factor amplitude $F_{P H}^{o}$. Aside from anomalous-scattering effects, there are three principal reasons for this: errors in measurement and scaling, errors in the heavy-atom model, and lack of isomorphism between native and derivative structures. The purpose of this section is to analyze these sources of error. Once we have done this, we will be able to write $F_{P H}^{o}$ and $F_{P H}^{c}$ in terms of variables which have known probability distributions. This will allow us to write an expression for the native-phase probability distribution.

\section{Errors in measurement}

The most obvious errors in $(1 a)$ are the errors in measuring and scaling of native and derivative structure-factor amplitudes. We assume here that $\sigma_{P}$ and $\sigma_{P H}$, the uncertainties in measurement of native and derivative structure-factor amplitudes, respectively, are known (e.g. from comparison of intensities of symmetry-related reflections). Assuming further that

(C) 1987 International Union of Crystallography 
the errors in measurement have a Gaussian distribution, we may express the probability of measuring a value of the derivative structure-factor amplitude between $F_{P H}^{o}$ and $F_{P H}^{o}+\mathrm{d} F_{P H}^{o}$, given that the true derivative structure-factor amplitude is $F_{P H}$ :

$$
\begin{aligned}
& P\left(F_{P H}^{o} \mid F_{P H}\right) \mathrm{d} F_{P H}^{o} \\
& \quad \propto \exp \left[-\left(F_{P H}^{o}-F_{P H}\right)^{2} / 2 \sigma_{P H}^{2}\right] \mathrm{d} F_{P H}^{o} .
\end{aligned}
$$

An analogous probability distribution may be written for the observed native structure-factor amplitude.

\section{Errors in the heavy-atom model}

A major uncertainty in $F_{P H}^{c}$, the calculated derivative structure-factor amplitude, is the error in the heavy-atom model leading to the estimate $f_{\mathbf{H}}$ of the heavy-atom structure factor. Let $\mathbf{F}_{\mathbf{H}}$ denote the true heavy-atom structure factor, define the 'residual heavy-atom structure factor' $\boldsymbol{\eta}$ as the difference between the true and estimated values of the heavyatom structure factor: $\boldsymbol{\eta} \equiv \mathbf{F}_{\mathbf{H}}-\mathbf{f}_{\mathbf{H}}$ (see Fig. 1 and Table 1). Then, if the native and derivative structures are perfectly isomorphous, the true native $\mathbf{F}_{\mathbf{p}}$ and derivative $\mathbf{F}_{\mathbf{P H}}$ structure factors are related by $\mathbf{F}_{\mathbf{P H}}=$ $\mathbf{F}_{\mathbf{P}}+\mathbf{f}_{\mathbf{H}}+\boldsymbol{\eta}$. We will assume that $\mathbf{f}_{\mathbf{H}}$ and $\boldsymbol{\eta}$ are not correlated, which will generally be the case unless incorrect sites have been included in the heavy-atom model.

As long as there are several minor heavy-atom sites not included in the heavy-atom model (as will generally be the case), the probability distribution for $\eta$ will be nearly a two-dimensional Gaussian distribution (Wilson, 1949). Defining $\eta \equiv\left(\eta_{x}, \eta_{y}\right)$, we may therefore write that, for acentric reflections, the probability that $\eta_{x}$ is between $\eta_{x}$ and $\eta_{x}+\mathrm{d} \eta_{x}$, and that $\eta_{y}$ is between $\eta_{y}$ and $\eta_{y}+\mathrm{d} \eta_{y}$ is

$$
\begin{aligned}
P(\boldsymbol{\eta}) \mathrm{d}^{2} \boldsymbol{\eta} & \equiv P\left(\eta_{x}, \eta_{y}\right) \mathrm{d} \eta_{x} \mathrm{~d} \eta_{y} \\
& \propto \exp \left[-\left(\eta_{x}^{2}+\eta_{y}^{2}\right) / H^{2}\right] \mathrm{d} \eta_{x} \mathrm{~d} \eta_{y}
\end{aligned}
$$

where the mean-square value of the residual heavyatom structure-factor amplitude $\left\langle|\eta|^{2}\right\rangle$ is $H^{2}$. For cen-

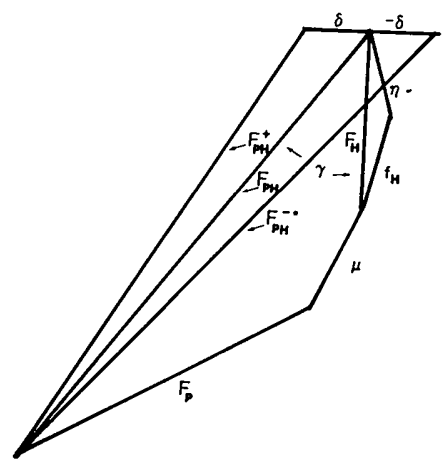

Fig. 1. The relationship of structure factors and other quantities

\begin{tabular}{|c|c|c|}
\hline Variable & Symbol & $\begin{array}{l}\text { Observed } \\
\text { quantity } \\
\text { (if any) }\end{array}$ \\
\hline $\begin{array}{l}\text { Native structure factor } \\
\text { and phase }\end{array}$ & $\mathbf{F}_{\mathbf{P}} \equiv F_{P} \exp (i \varphi)$ & $F_{P}^{o}$ \\
\hline $\begin{array}{l}\text { Derivative structure factors } \\
\text { (elements of a Friedel pair) }\end{array}$ & $\mathbf{F}_{\mathbf{P H}}^{+}, \mathbf{F}_{\mathbf{P H}}^{-}$ & $\bar{F}_{P H}^{o}$ \\
\hline $\begin{array}{l}\text { Average derivative structure } \\
\text { factor }\end{array}$ & $\overline{\mathbf{F}}_{\mathbf{P H}}$ & \\
\hline $\begin{array}{l}\text { Anomalous difference } \\
\text { Heavy-atom structure factor }\end{array}$ & $\Delta_{P H}$ & $\Delta_{P H}^{o}$ \\
\hline $\begin{array}{l}\text { non-anomalous part } \\
\text { anomalous part }\end{array}$ & $\underset{\mathbf{\delta}}{\mathbf{F}_{\mathbf{H}}}$ & \\
\hline $\begin{array}{l}\text { Estimated heavy-atom structure } \\
\text { factor (non-anomalous part) }\end{array}$ & $\mathbf{f}_{\mathbf{H}}$ & \\
\hline $\begin{array}{l}\text { Residual heavy-atom structure } \\
\text { factor (non-anomalous part) }\end{array}$ & $\eta$ & \\
\hline $\begin{array}{l}\text { Lack-of-isomorphism structure } \\
\text { factor }\end{array}$ & $\boldsymbol{\mu}$ & \\
\hline \multicolumn{3}{|c|}{ Basic relationships } \\
\hline \multicolumn{3}{|c|}{$\mathbf{F}_{\mathbf{P H}} \equiv \mathbf{F}_{\mathbf{P}}+\boldsymbol{\mu}+\mathbf{F}_{\mathbf{H}}$} \\
\hline \multicolumn{3}{|c|}{$\mathbf{F}_{\mathbf{H}} \equiv \mathbf{f}_{\mathbf{H}}+\boldsymbol{\eta}$} \\
\hline \multicolumn{3}{|c|}{$\mathbf{F}_{\mathbf{P H}}^{+} \equiv \overline{\mathbf{F}}_{\mathbf{P H}}+\boldsymbol{\delta}$} \\
\hline \multicolumn{3}{|c|}{$\mathbf{F}_{\mathbf{P H}}^{-*} \equiv \overline{\mathbf{F}}_{\mathbf{P H}}-\delta$} \\
\hline
\end{tabular}
discussed in the text. Definitions of all symbols are summarized in Table 1.
Table 1. Summary of principal variables

tric reflections, which can have only one of two possible phases, the probability distribution for $\boldsymbol{\eta}$ is a one-dimensional Gaussian with the same meansquare amplitude $H^{2}$ (Wilson, 1949). Denoting the residual heavy-atom structure factor by the scalar $\eta$, we have

$$
P(\eta) \mathrm{d} \eta \propto \exp -\left(\eta^{2} / 2 H^{2}\right) \mathrm{d} \eta .
$$

Notice that although the mean-square values $H^{2}$ are equal in the acentric and centric cases, the denominators of the exponentials in $(3 a)$ and $(3 b)$ differ by a factor of two: this is the difference between one-dimensional and two-dimensional Gaussian distributions with equal mean-square values.

\section{Lack of isomorphism}

Another major error in the calculated derivative structure-factor amplitude $F_{P H}^{c}$ is the effect of nonisomorphism between native and derivative structures. We define the lack-of-isomorphism structure factor $\boldsymbol{\mu}$ as the difference between true native and derivative structure factors caused by all sources except heavy-atom substitution in the derivative structure.

$$
\boldsymbol{\mu} \cong \mathbf{F}_{\mathbf{P H}}-\left(\mathbf{F}_{\mathbf{P}}+\mathbf{F}_{\mathbf{H}}\right),
$$

where $\mathbf{F}_{\mathbf{P H}}, \mathbf{F}_{\mathbf{P}}$, and $\mathbf{F}_{\mathbf{H}}$ are defined above.

The probability distribution for the lack-ofisomorphism structure factor $\mu$ is, like that for the residual heavy-atom structure factor $\eta$, very nearly a two-dimensional Gaussian for acentric reflections and a one-dimensional Gaussian for centric reflections, as is shown in the Appendix. We can therefore 
express the probability distribution for the lack-ofisomorphism structure factor for centric reflections as

$$
P(\mu) \mathrm{d} \boldsymbol{\mu} \propto \exp \left(-\mu^{2} / 2 M^{2}\right) \mathrm{d} \mu
$$

where $\left\langle\mu^{2}\right\rangle=M^{2}$. For acentric reflections a nearly identical treatment may be applied. Defining $\boldsymbol{\mu}=$ $\left(\mu_{x}, \mu_{y}\right)$, we may write

$$
\begin{aligned}
P(\boldsymbol{\mu}) \mathrm{d}^{2} \boldsymbol{\mu} & \equiv P\left(\mu_{x}, \mu_{y}\right) \mathrm{d} \mu_{x} \mathrm{~d} \mu_{y} \\
& \propto \exp \left[-\left(\mu_{x}^{2}+\mu_{y}^{2}\right) / M^{2}\right] \mathrm{d} \mu_{x} \mathrm{~d} \mu_{y}
\end{aligned}
$$

where the mean value $\left\langle|\boldsymbol{\mu}|^{2}\right\rangle$ is again $\boldsymbol{M}^{2}$.

We have now obtained the probability distributions for the principal sources of error in $(1 a)$ : the errors in measurement of native and derivative structurefactor amplitudes, the errors in the heavy-atom model and the lack of isomorphism between native and derivative structures. The errors from these sources are essentially uncorrelated with each other and with the native and derivative structure factors so that we may treat them all as independent variables in calculating a probability distribution for the native phase $\varphi$. Note that given $\mathbf{F}_{\mathbf{P}}, \mathbf{f}_{\mathbf{H}}, \boldsymbol{\eta}$ and $\boldsymbol{\mu}$ we can write an expression for the derivative structure factor $\mathbf{F}_{\mathbf{P H}}$ (Fig. 1):

$$
\mathbf{F}_{\mathbf{P H}}=\mathbf{F}_{\mathbf{P}}+\boldsymbol{\mu}+\mathbf{f}_{\mathbf{H}}+\boldsymbol{\eta} .
$$

\section{Calculation of the native-phase probability distribution}

A straightforward application of statistical methods may now be used to calculate the native-phase probability distribution. The central element in this calculation is the relationship known as Bayes' rule (Hamilton, 1964), which we illustrate now. Suppose we have some knowledge of the probability distribution for a variable (from a previous experiment), so that before making a new measurement we may write ( $a$ priori) that the probability it is between $x$ and $x+\mathrm{d} x$ is $P_{0}(x) \mathrm{d} x$. Now we make a new measurement of this variable, call its value $y$. Suppose that we know enough about our procedure to say that if the parent value of $y$ is $x$, then the probability that we would measure $y$ between $y$ and $y+\mathrm{d} y$ is $P(y \mid x) \mathrm{d} y$. Bayes' rule states that we may calculate a new probability distribution for $x$ based on the a priori information $P_{0}(x)$, the measurement $y$, and the probability that we would have measured $y$ if $x$ were its parent value:

$$
P(x)=P_{0}(x) P(y \mid x) / \int_{\text {all } x} P_{0}(x) P(y \mid x) \mathrm{d} x
$$

or, more simply,

$$
P(x) \propto P_{0}(x) P(y / x) .
$$

In the present case, we are interested only in the probability distribution for $\varphi$, the native phase. In order to obtain this, however, we must first calculate the joint probability distribution for $\varphi, F_{B} \eta$ and $\mu$.
Then we will integrate this joint probability distribution over all values of $F_{P} \boldsymbol{\eta}$ and $\boldsymbol{\mu}$, in order to obtain the probability distribution for $\varphi$. The set of variables $\varphi, F_{P}, \eta$ and $\mu$ corresponds to the single variable $x$ in the preceding example.

We have a priori information about some of the fundamental variables in this calculation. Consider first an acentric reflection. If there is phase probability information from other sources, denote this a priori distribution by $P_{0}(\varphi)$. We assume that these other sources are independent so that the a priori phase probability distribution does not depend on any of the variables of current interest. An a priori probability distribution for the native structure-factor amplitude is also known (Wilson, 1949),

$$
P_{0}\left(F_{P}\right) \propto F_{P} \exp \left(-F_{P}^{2} / \Sigma\right)
$$

where $\Sigma$ is the mean-square native structure-factor amplitude at the appropriate resolution. In $(3 a)$ and (4a) we have a priori probability distributions for $\boldsymbol{\eta}$ and $\mu$. Before making any measurements of $F_{P}^{o}$ and $F_{P H}^{o}$, then, we may write that the joint probability distribution for $\varphi, F_{P}, \eta$ and $\mu$ is

$$
P_{0}\left(\varphi, F_{P}, \eta, \mu\right) \propto P_{0}(\varphi) P_{0}\left(F_{P}\right) P(\eta) P(\mu) .
$$

Now, if we apply Bayes' rule after making measurements $F_{P}^{o}$ and $F_{P H}^{o}$ of the native and derivative structure-factor amplitudes, we may use (2) and the analogous equation for $F_{P}^{o}$ to express $P$ :

$$
\begin{aligned}
P\left(\varphi, F_{P}, \boldsymbol{\eta}, \boldsymbol{\mu}\right) \propto & P_{0}\left(\varphi, F_{P}, \eta, \mu\right) \\
& \times P\left(F_{P}^{o} \mid F_{P}\right) P\left(F_{P H}^{o} \mid F_{P H}\right)
\end{aligned}
$$

where $F_{P H} \equiv\left|\mathbf{F}_{\mathbf{P H}}\right|$ is calculated using (5). Finally, to obtain the probability distribution for $\varphi$ alone, we may integrate (10) over all possible values of $F_{P}, \boldsymbol{\eta}, \boldsymbol{\mu}$ :

$$
P(\varphi) \propto \int P\left(\varphi, F_{P}, \boldsymbol{\eta}, \boldsymbol{\mu}\right) \mathrm{d} F_{P} \mathrm{~d}^{2} \boldsymbol{\eta} \mathrm{d}^{2} \boldsymbol{\mu}
$$

or, using (2), (3), (4b) and (8),

$$
\begin{aligned}
P(\varphi) \propto & P_{0}(\varphi) \int \exp \left[-F_{P}^{2} / \Sigma-|\eta|^{2} / H^{2}\right. \\
& -|\boldsymbol{\mu}|^{2} / M^{2}-\left(F_{P}-F_{P}^{o}\right)^{2} / 2 \sigma_{P}^{2} \\
& \left.-\left(F_{P H}-F_{P H}^{o}\right)^{2} / 2 \sigma_{P H}^{2}\right] F_{P} \mathrm{~d} F_{P} \mathrm{~d}^{2} \eta \mathrm{d}^{2} \boldsymbol{\mu}
\end{aligned}
$$

where $F_{P H}$ is calculated from (5). Equation (12) is an exact expression which includes all the information discussed here relating to the probability distribution for the native phase $\varphi$. If we assume further that $\sigma_{P}, \sigma_{P H},|\boldsymbol{\eta}|$ and $|\boldsymbol{\mu}|$ as well as $\left|\mathbf{f}_{\mathbf{H}}\right|$ are small relative to $F_{P}^{o}$ and $F_{P H}^{o},(12)$ may be integrated using firstorder approximations to yield an approximate phase probability distribution for acentric reflections:

$$
\begin{aligned}
P(\varphi) \propto & \exp \left\{-\left(F_{P H}^{o}-F_{P H}^{c}\right)^{2}\right. \\
& \left.\times\left[H^{2}+M^{2}+2 \sigma_{P}^{2}+2 \sigma_{P H}^{2}\right]^{-1}\right\} P_{0}(\varphi),
\end{aligned}
$$


where $F_{P H}^{c}$ is evaluated using $(1 b)$. Except for the $a$ priori probability distribution for the native phase $P_{0}(\varphi)$, this is equivalent to the formula given by Blow $\&$ Crick (1959), as shown in (1a), where $E^{2}$ is given by

$$
E^{2}=E_{\mathrm{ACENT}}^{2}=H^{2} / 2+M^{2} / 2+\sigma_{P}^{2}+\sigma_{P H}^{2} .
$$

Our procedure for calculating the phase probability distribution, which begins from very basic assumptions, has yielded the same result as this much earlier work except that the contributions of various sources to $E^{2}$ are now separated into defined components.

An analogous procedure may be used to obtain an expression for the phase probability distribution for centric reflections:

$$
\begin{aligned}
P(\varphi) \propto & P_{0}(\varphi) \int \exp \left[-F_{P}^{2} / 2 \Sigma-\eta^{2} / 2 H^{2}\right. \\
& -\mu^{2} / 2 M^{2}-\left(F_{P}-F_{P}^{o}\right)^{2} / 2 \sigma_{P}^{2} \\
& \left.-\left(F_{P H}-F_{P H}^{o}\right)^{2} / 2 \sigma_{P H}^{2}\right] \mathrm{d} F_{P} \mathrm{~d} \eta \mathrm{d} \mu
\end{aligned}
$$

which may be integrated, using the same approximations as above, to yield

$$
\begin{aligned}
P(\varphi) \propto & \exp \left\{-\left(F_{P H}^{o}-F_{P H}^{c}\right)^{2}\right. \\
& \left.\times\left[2 H^{2}+2 M^{2}+2 \sigma_{P}^{2}+2 \sigma_{P H}^{2}\right]^{-1}\right\} P_{0}(\varphi) .
\end{aligned}
$$

This expression is identical to that for acentric reflections except that $E^{2}$ for centric reflections is

$$
E_{\mathrm{CENT}}^{2}=H^{2}+M^{2}+\sigma_{P}^{2}+\sigma_{P H}^{2} .
$$

That is, aside from errors in measurement, the centric $E^{2}$ is twice the acentric $E^{2}$. This difference is entirely due to the difference between the expressions for one-dimensional and two-dimensional Gaussian distributions with equal mean-square values [e.g. $(3 a)$ and $(3 b)]$.

Calculation of the phase probability distribution in the presence of anomalously scattering atoms in the derivative structure

In order to calculate the phase probability distribution in the presence of anomalously scattering heavy atoms in the derivative structure, we need expressions for the average derivative structure factor $\overline{\mathbf{F}}_{\mathbf{P H}}$ and the anomalous difference $\Delta_{P H}$ (North, 1965). Since anomalous differences are rarely measured with an accuracy much better than the typical values of the anomalous differences, relatively crude approximations will suffice here. If we assume that the heavyatom structure-factor amplitude $\left|\mathbf{F}_{\mathbf{H}}\right|$ is small relative to the native structure-factor amplitude $\left|\mathbf{F}_{\mathbf{P}}\right|$, the average derivative structure-factor amplitude $\vec{F}_{P H}$ and the anomalous difference $\Delta_{P H}$ are given approximately by (see the Appendix and North, 1965):

$$
\begin{aligned}
& \bar{F}_{P H} \equiv \frac{1}{2}\left(\left|\mathbf{F}_{\mathbf{P H}}^{+}\right|+\left|\mathbf{F}_{\mathbf{P H}}^{-}\right|\right) \doteq F_{P H}, \\
& \Delta_{P H} \equiv \frac{1}{2}\left(\left|\mathbf{F}_{\mathbf{P H}}^{+}\right|-\left|\mathbf{F}_{\mathbf{P H}}^{-}\right|\right) \doteq-1 / \kappa F_{H} \sin \gamma
\end{aligned}
$$

where $\gamma$ is the angle between $\mathbf{F}_{\mathbf{H}}$ and $\mathbf{F}_{\mathbf{P H}}^{+}, \mathbf{F}_{\mathbf{P H}}$ is from (5), and $\kappa$ is the ratio of real to anomalous scattering for the heavy atoms. Notice that the anomalous difference is relatively insensitive to lack of isomorphism.

An expression analogous to (12) for the phase probability distribution when anomalous differences have been measured is:

$$
\begin{aligned}
P(\varphi) \propto & P_{0}(\varphi) \int \exp \left[-F_{P}^{2} / \Sigma-|\eta|^{2} / H^{2}-|\boldsymbol{\mu}|^{2} / M^{2}\right. \\
& -\left(F_{P}-F_{P}^{o}\right)^{2} / 2 \sigma_{P}^{2}-\left(\bar{F}_{P H}-\bar{F}_{P H}^{o}\right)^{2} / 2 \sigma_{P H}^{2} \\
& \left.-\left(\Delta_{P H}-\Delta_{P H}^{o}\right)^{2} / 2 \sigma_{\text {ANO }}^{2}\right] F_{P} \mathrm{~d} F_{P} \mathrm{~d}^{2} \eta \mathrm{d}^{2} \mu
\end{aligned}
$$

where $\bar{F}_{P H}$ and $\Delta_{P H}$ are calculated using (18) and (19), $\sigma_{\text {ANO }}$ is the uncertainty in the anomalous difference, and the integral is over all possible values of $F_{B}, \boldsymbol{\eta}$ and $\boldsymbol{\mu}$. With the same approximations which led to (12), (20) may be integrated to yield an approximate phase probability distribution when anomalous differences have been measured:

$$
\begin{aligned}
P(\varphi) \propto & \exp \left\{-\left(\bar{F}_{P H}^{o}-\bar{F}_{P H}^{c}\right)^{2}\right. \\
& \times\left[H^{2}+M^{2}+2 \sigma_{P}^{2}+2 \sigma_{P H}^{2}\right]^{-1} \\
& \left.-\left(\Delta_{P H}^{o}-\Delta_{P H}^{c}\right)^{2}\left[H^{2} / \kappa^{2}+2 \sigma_{A N O}^{2}\right]^{-1}\right\} P_{0}(\varphi)
\end{aligned}
$$

where $\bar{F}_{P H}^{c}$ and $\Delta_{P H}^{c}$ are calculated using (18) and (19).

The first term in the exponent of (21) is identical to the exponent in (13). The second term is due to the effects of anomalous scattering and is identical to that given by North (1965) except that $E_{\text {ANO is }}^{2}$ given by the expression

$$
E_{\mathrm{ANO}}^{2}=\left(H^{2} / 2 \kappa^{2}\right)+\sigma_{\mathrm{ANO}}^{2} .
$$

Notice that $E_{\text {ANO }}^{2}$ does not depend on the lack of isomorphism $\left(M^{2}\right)$.

\section{Estimation of $\boldsymbol{E}_{\mathrm{ACENT}}^{\mathbf{2}}, \boldsymbol{E}_{\mathrm{CENT}}^{\mathbf{2}}$ and $\boldsymbol{E}_{\mathrm{ANO}}^{\mathbf{2}}$}

Equations (13), (16) and (19) require estimates of $E_{\mathrm{ACENT}}^{2}, E_{\mathrm{CENT}}^{2}$ and $E_{\mathrm{ANO}}^{2}$, which are not available directly from measurements. To develop a method for estimating them we first calculate the expected value of the centric, acentric and anomalous meansquare lack-of-closure residuals, evaluated in the usual fashion (Blow \& Crick, 1959; Ten Eyck \& Arnone, 1976), assuming that the native phase $\varphi$ is known precisely. Using the same first-order approximations as above, it may be shown, using $(3 b),(4 a)$ and (5), that the mean-square value of the centric lack-of-closure residual is

$$
\begin{array}{r}
\left\langle\left(F_{P H}^{o}-F_{P H}^{c}\right)^{2}\right\rangle \doteq H^{2}+M^{2}+\left\langle\sigma_{P}^{2}\right\rangle+\left\langle\sigma_{P H}^{2}\right\rangle \\
\text { (centric). }
\end{array}
$$

If we assume once again that the native phase is known, the expected values of the acentric and 
anomalous lack-of-closure residuals are given, using $(3 a),(4 b),(5),(A 16),(18)$ and $(19)$, by

$$
\left\langle\left(F_{P H}^{o}-F_{P H}^{c}\right)^{2}\right\rangle \doteq H^{2} / 2+M^{2} / 2+\left\langle\sigma_{P}^{2}\right\rangle+\left\langle\sigma_{P H}^{2}\right\rangle
$$

(acentric)

and

$$
\left\langle\left(\Delta_{\mathrm{PH}}^{o}-\Delta_{P H}^{c}\right)^{2}\right\rangle \doteq H^{2} /\left(2 \kappa^{2}\right)+\left\langle\sigma_{\mathrm{ANO}}^{2}\right\rangle
$$

where the constant $\kappa$ is defined above.

Comparing (23)-(25) with (14), (17) and (22), it is clear that the appropriate values of $E^{2}$ for centric, acentric and anomalous differences in the formulations of Blow \& Crick (1959) and North (1965) are the usual mean-square lack-of-closure residuals calculated using the correct native phases. It must be noted that centric and acentric reflections are to be treated separately, however.

The standard methods of estimating the lack-ofclosure errors (Blow \& Crick, 1959; Ten Eyck \& Arnone, 1976) rely on the values of the lack-of-closure residuals evaluated at the 'best' or 'most probable' native phases. If the native phases are not known with certainty, then this procedure will yield inaccurate values of the lack-of-closure errors (Blow \& Matthews, 1973). Since a probability distribution for the phase is available [(21), for example], improved estimates of the lack-of-closure errors may be obtained simply by averaging the standard lack-ofclosure residuals over all native phases, weighting by the probability that each phase is correct. We therefore define the 'averaged' lack-of-closure residuals $\bar{E}_{\text {ACENT, }}^{2} \bar{E}_{\text {CENT }}^{2}$ and $\bar{E}_{\text {ANO }}^{2}$ as follows:

$$
\begin{aligned}
\bar{E}_{\mathrm{CENT}}^{2} & \equiv\left\langle\sum_{j=1}^{2} P\left(\varphi_{j}\right)\left[F_{P H}^{o}-F_{P H}^{c}\left(\varphi_{j}\right)\right]^{2}\right\rangle, \\
\bar{E}_{\mathrm{ACENT}}^{2} & \equiv\left\langle\int P(\varphi)\left[\bar{F}_{P H}^{o}-\bar{F}_{P H}^{c}(\varphi)\right]^{2} \mathrm{~d} \varphi\right\rangle, \\
\bar{E}_{\mathrm{ANO}}^{2} & \equiv\left\langle\int P(\varphi)\left[\Delta_{P H}^{o}-\Delta_{P H}^{c}(\varphi)\right]^{2} \mathrm{~d} \varphi\right\rangle,
\end{aligned}
$$

where the normalized phase probability distribution $P(\varphi)$ includes all information available and the angle brackets indicate an average over all appropriate reflections in a given range of resolution.

\section{Estimation of the mean-square residual heavy-atom and lack-of-isomorphism structure factors}

Based on the preceding section, we can expect that the averaged lack-of-closure residuals defined by (26)-(28) will be reasonable estimates of $E_{\mathrm{ACENT}}^{2}$, $E_{\text {CENT }}^{2}$ and $E_{\text {ANO. Also, if we recall (14), (17) and }}^{2}$ (22), it is evident that the averaged lack-of-closure residuals may be used to estimate the mean-square residual heavy-atom structure factor $\left(H^{2}\right)$ and the mean-square lack-of-isomorphism structure factor $\left(M^{2}\right)$ at a given resolution. From (14), (17), (26) and (27) we have

$$
\begin{aligned}
& H^{2}+M^{2} \doteq \bar{E}_{\mathrm{CENT}}^{2}-\left\langle\sigma_{P}^{2}\right\rangle-\left\langle\sigma_{P H}^{2}\right\rangle, \\
& H^{2}+M^{2} \doteq 2\left(\bar{E}_{\mathrm{ACENT}}^{2}-\left\langle\sigma_{P}^{2}\right\rangle-\left\langle\sigma_{P H}^{2}\right\rangle\right)
\end{aligned}
$$

where the angle brackets indicate an average over all centric or acentric reflections in the appropriate range of resolution. The sum of $H^{2}$ and $M^{2}$ may therefore be obtained either from the averaged centric lack-ofclosure residual or the averaged acentric lack-ofclosure residual.

The average anomalous lack-of-closure residual $\bar{E}_{\text {ANO }}^{2}$ depends on $H^{2}$ but not on $M^{2}$, so that if anomalous differences have been measured, separate estimates of $H^{2}$ and $M^{2}$ may be obtained. Using (22) and (28), we may write

$$
H^{2} \doteq\left\langle\kappa^{2}\right\rangle\left(\bar{E}_{\mathrm{ANO}}^{2}-\left\langle\sigma_{\mathrm{ANO}}^{2}\right\rangle\right),
$$

where the values of $\kappa$ are the same as those used in (21). Since the value of $\kappa$ is typically greater than unity, it is clear that estimates of $H^{2}$ obtained from (31) will generally be fairly coarse. Notice that the estimate of $H^{2}$ does require an accurate estimate of the uncertainties in measurement of the anomalous differences $\left\langle\sigma_{\mathrm{ANO}}^{2}\right\rangle$.

\section{Discussion}

We have shown here that, with reasonable approximations, the phase probability distributions given by Blow \& Crick (1959) and by North (1965) and Matthews (1966) can be derived from basic premises. Blow \& Crick (1959), citing empirical evidence, assumed that the errors in observed and calculated derivative structure-factor amplitudes were distributed in a Gaussian fashion. Approximations similar to those we have used led them to $(1 a)$. On the basis of a detailed analysis of errors, we have found that the assumption of Gaussian distributions is very reasonable, justifying ( $1 a)$. More importantly, the present analysis has yielded a straightforward interpretation of the $E^{2}$ for centric, acentric and anomalous differences [(14), (17) and (22)] and an improved method of estimating them from the averaged lack-of-closure residuals [(26)-(28)].

Equations $(1 a),(13),(16)$ and (21) are all approximate phase probability distributions. Given our assumptions, however, (12), (15) and (20) are exact expressions. Nearly any desired degree of accuracy in the phase probability distributions can be obtained by a suitably precise evaluation of these expressions. Green (1979) has analyzed expressions similar to (12) with few approximations, and found that, in cases where lack of isomorphism was not severe, the resulting phase probability distribution and that obtained using (13) were quite similar. We have also carried out numerical integrations of (12) and find that the resultant phase probability distribution is given very precisely by (13) when $\left|\mathbf{f}_{\mathbf{H}}\right|,|\boldsymbol{\eta}|$ and $|\boldsymbol{\mu}|$ are all less than $20 \%$ of $F_{P}^{o}$.

An error far more serious than the approximate evaluation of (12) [or of (15) or (20)] is that caused by using inaccurate values of the lack-of-closure 
errors. A factor-of-two change in the lack-of-closure error in $(1 a)$ is sufficient to convert a probability distribution with a 'figure of merit' of 0.45 into one with a figure of merit of 0.07 , an error far greater than that typically caused by using $(1 a)$ instead of (12). This is why we have focused attention on the evaluation of these lack-of-closure errors and why we suggest the use of the averaged lack-of-closure residuals $[(26)-(28)]$ for this purpose.

In order to determine the effects of this new procedure, we have calculated lack-of-closure residuals using data from a model native structure and from model derivative structures which included heavyatom substitution and which were not completely isomorphous with the native structure. Table 2 compares the estimates of the mean-square lack-ofclosure residuals obtained from (26)-(28) with their expected values from (14), (17) and (22) and with the conventional lack-of-closure residuals for a case with one derivative and a mean figure of merit of $0 \cdot 47$. In this test, lack-of-closure residuals calculated using the 'best' or 'most probable' phases were seriously underestimated, even for centric reflections. The averaged lack-of-closure residuals, however, were reasonable estimates of the true lack-of-closure errors, even in this case with one derivative. Also notice that, as predicted by (23) and (24), the meansquare centric lack-of-closure residual is essentially twice the mean-square acentric lack-of-closure residual.

The mean-square residual heavy-atom structurefactor amplitude $\left(H^{2}\right)$ and the mean-square lack-ofisomorphism structure-factor amplitude $\left(M^{2}\right)$ may be estimated from the averaged lack-of-closure residuals using (29)-(31), if the anomalous differences have been measured and uncertainties in these measurements are known. In the example in Table 2, anomalous differences were calculated, and in Table 3 are presented the estimates of $H^{2}$ and $M^{2}$ calculated from (29)-(31) with the data of Table 2. As noted earlier, the separate estimation of $H^{2}$ and $M^{2}$ depends on an accurate estimate of the uncertainty in measurement of the anomalous differences. These estimates were available in the present example, but are not available in all cases. When $H^{2}$ and $M^{2}$ can be accurately estimated, they may be used to determine when the derivative is 'solved', and all remaining differences between native and derivative structures are due to errors in measurement and lack of isomorphism.

As noted by Einstein (1977), our assumption that all a priori sources of phase information are independent of the present calculation is not always strictly correct. One set of native structure factors is often used in conjunction with each of several derivative sets of structure-factor amplitudes. Consequently, the phase probability distributions for the various derivative-native combinations are not independent.

\section{Table 2. Estimation of lack-of-closure errors using} one derivative

Native and derivative structure-factor amplitudes were calculated from a model for 407 acentric and 56 centric reflections from $2 \cdot 0$ to $2 \cdot 1 \AA$ resolution. The models were based on the partially refined structure of melittin form II crystals (space group C222. liger \& Eisenberg, 1982). In order to introduce non-isomorphism between the derivative structure and the native structure, the coordinates of six protein atoms were moved a r.m.s. distance of $0.8 \AA$ in the derivative structure. The actual mean-square "lack-ofisomorphism' structure-factor amplitude $\left(\mu^{2}\right)$ in Table 3 was calculated using equation $(A 3)$ for centric reflections and the analogous expression for acentric reflections. The heavy-atom contribution to the derivative structure factor was calculated using five arbitrarily placed heavy-atom sites of equal occupancy. The magnitudes of the native and derivative structure factors were then calculated, and, after addition of a small random 'observational error', these amplitudes were used as the 'observed' native and derivative structure-factor amplitudes. The r.m.s. native structure-factor amplitude is 140 , the r.m.s. 'observational' uncertainty in the native structurefactor amplitudes is 1.5 , and the r.m.s. uncertainty in the derivative structure-factor amplitudes and anomalous differences is also $1 \cdot 5$.

In calculating phase probability distributions and lack-of-closure residuals, three of the five heavy-atom sites were included with the correct occupancy in the calculation of the 'estimates' of the heavy-atom structure factors $\left(\mathbf{f}_{\mathbf{H}}\right)$. The 'actual' mean-square residual heavy-atom structure-factor amplitude in Table 3 is the r.m.s. heavy-atom structure-factor amplitude due to the two sites not included in the model. The expected values of the residuals were calculated using the data in Table 3 . The lack-of-closure residuals averaged over all phases were determined using equations (26)-(28). Those estimated at the 'true', 'best' or 'most probable' phases were calculated using the same equations, setting $P(\varphi)$ equal to zero except at the 'true', 'best' or 'most probable' phase.

For each of the three methods of estimating lack-of-closure residuals, phase probability distributions were evaluated using equations (16) and (21) which themselves require estimates of the lack-of-closure errors. Therefore, a procedure designed to simulate a real situation was followed. For each method, the average value of $\left(F_{P H}^{o}-F_{P}^{o}\right)^{2}$ over all centric reflections was used as a starting value of the centric lack-of-closure error, and the average value of $\left(\Delta_{P H}^{o}\right)^{2}$ over all acentric reflections was used as a starting value of the anomalous lack-of-closure error. The acentric lack-of-closure error was always taken to be half the centric lack-of-closure error. Phase probability distributions were calculated and new estimates of the lack-of-closure errors were obtained from the corresponding lack-of-closure residuals. The new estimate of the centric lack-ofclosure error was the weighted average of the centric lack-of-closure residual and twice the acentric lack-of-closure residual. This cycle was repeated once more and the residuals listed were found.

In calculating the lack-of-closure residuals, only reflections which satisfied the relation

$$
F_{P H}^{o}>4\left(\sigma_{P}^{2}+\sigma_{P H}^{2}+H^{2}+M^{2}\right)
$$

were included. This criterion resulted in the rejection of $21 \%$ of the centric and $12 \%$ of the acentric reflections. The mean figure of merit for the test was 0.47 .

Native phase used to estimate lack-of-closure Mean-square lack-of-closure residual residuals Centric Acentric Anomalous

Expected values

[equations (14), (17), (22)]

True $\varphi$

Averaged over $\varphi$

'Best' $\varphi$

'Most probable' $\varphi$
304

270

330

210

210
154

140

160

80

80
$3 \cdot 5$

3.5

$3 \cdot 4$

1.4 
Table 3. Estimation of $H^{2}$ and $M^{2}$ from the data in Table 2

The r.m.s. residual heavy-atom structure-factor amplitude $\left(H^{2}\right)$ was estimated from the data in Table 2 using equation (22). The value of $\kappa$ was 5.8 in all cases. The r.m.s. lack-of-isomorphism structure-factor amplitude $\left(M^{2}\right)$ was calculated from $H^{2}$ and equations (14) and (17).

Actual value

Estimates from the single derivative

$\begin{array}{cc}H^{2} & M^{2} \\ 90 & 210 \\ 80 & 260\end{array}$

However, as long as the variances $\sigma_{P}^{2}$ of the observed native structure-factor amplitudes are small relative to the overall error $\left(\frac{1}{2} H^{2}+\frac{1}{2} M^{2}+\sigma_{P H}+\sigma_{P}^{2}\right)$, the errors in measurement of the native structure-factor amplitudes will not greatly affect the phase probability distribution.

Caution should be exercised when the various derivatives share heavy-atom sites. A substantial correlation between the phase probability distributions obtained for various derivatives may occur when there are unknown heavy-atom sites in common in the various derivatives. In this case, the residual heavyatom structure factor $\boldsymbol{\eta}$ is not independent in the various derivatives and therefore the phase probability distributions based on the various derivatives are not independent. Of course, it is difficult to know when two derivatives share unknown heavy-atom sites, but it seems likely that, in cases where known heavy-atom sites are in common in two derivatives, unknown sites are also shared. A similar argument applies to the correlation between the lack-of-isomorphism structure factors $\boldsymbol{\mu}$ in various derivatives which share heavy-atom sites.

A Fortran program (HEAVY) which incorporates the results described here may be obtained from the Protein Data Bank, Brookhaven National Laboratory, Upton, Long Island, New York 11973, USA.

We thank Drs S. Sheriff, L. Weissman, W. Smith and R. Sweet for helpful discussions. This work was supported by USPHS grant GM-31299. TCT was the recipient of a National Science Foundation Graduate Fellowship.

\section{APPENDIX}

Probability distribution for the lack-of-isomorphism structure factor

Consider a centric reflection. In this case, the native structure factor $F_{P}$ may be written as a scalar (Wilson, 1949).

$$
F_{P}=2 \sum_{j=1}^{N / 2} f_{j} \cos \left(2 \pi \mathbf{s} \cdot \mathbf{x}_{j}\right)
$$

where $f_{j}$ and $\mathbf{x}_{j}$ are the form factors and coordinates of atom $j, \mathbf{s} \equiv h \mathbf{a}^{*}+k \mathbf{b}^{*}+l \mathbf{c}^{*}$ is the position of this reflection in reciprocal space, and there are $N$ atoms in the unit cell. Then we may write an expression for the contribution $F_{P}^{\prime}$ of atoms present in the native structure to the derivative structure factor $F_{P H}$ :

$$
F_{P}^{\prime}=2 \sum_{j=1}^{N / 2} f_{j} \cos \left[2 \pi \mathbf{s} .\left(\mathbf{x}_{j}+\delta \mathbf{x}_{j}\right)\right]
$$

where atom $j$ has shifted position from $\mathbf{x}_{j}$ to $\mathbf{x}_{j}+\delta \mathbf{x}_{j}$ from native to derivative structures. An expression for the lack-of-isomorphism structure factor $\mu$ in this case is

$$
\mu=2 \sum_{j=1}^{N / 2} f_{j}\left\{\cos \left[2 \pi \mathbf{s} .\left(\mathbf{x}_{j}+\delta \mathbf{x}_{j}\right)\right]-\cos \left(2 \pi \mathbf{s} . \mathbf{x}_{j}\right)\right\}
$$

or, after rearrangement,

$$
\begin{aligned}
\mu= & 2 \sum_{j=1}^{N / 2} f_{j}\left\{\cos \left(2 \pi \mathbf{s} \cdot \mathbf{x}_{j}\right)\left[\cos \left(2 \pi \mathbf{s} . \delta \mathbf{x}_{j}\right)-1\right]\right. \\
& \left.-\sin \left(2 \pi \mathbf{s} . \mathbf{x}_{j}\right) \sin \left(2 \pi \mathbf{s} . \delta \mathbf{x}_{j}\right)\right\} .
\end{aligned}
$$

Now, using reasoning similar to that used by Wilson (1949), we note that $\cos \left(2 \pi \mathbf{s} . \mathbf{x}_{j}\right)$ and $\sin \left(2 \pi \mathbf{s} . \mathbf{x}_{j}\right)$ in $(A 4)$ vary in an essentially random fashion from -1 to 1 . Therefore, $\mu$ is the sum of many uncorrelated random variables with mean values of zero. According to the central-limit theorem (Hamilton, 1964), $\mu$ will be distributed in a Gaussian fashion with an expected value of zero and a mean-square value equal to the sum of the mean-square values of the random variables. This can be shown to be

$$
\left\langle\mu^{2}\right\rangle=4 \sum_{j=1}^{N / 2} f_{j}^{2}\left[1-\cos \left(2 \pi \mathbf{s} . \delta \mathbf{x}_{j}\right)\right] .
$$

If there are many terms in $(A 5)$ and the $\delta \mathbf{x}_{j}$ are randomly distributed, this expected mean-square value of $\mu$ will characterize all reflections at a given resolution (at other resolutions, the form factors $f_{i}$ will be different). This leads to $(4 a)$ and a similar argument leads to $(4 b)$.

It may be shown that in cases of extreme nonisomorphism (for example, native and derivative structures unrelated) the lack-of-isomorphism structure factor $\mu$ will be strongly correlated with both the native and derivative structure factors. When the magnitude of $\mu$ is small relative to the native structure-factor amplitude, however, $\mu$ is correlated with neither. To show this for centric reflections (as before, a nearly identical argument applies to acentric reflections), define the correlation $\alpha$ between $\mu$ and $F_{P}$ as the average value, over many reflections, of the product of $\mu$ and $F_{P}$, divided by the r.m.s. values of $\mu$ and $F_{P}$ :

$$
\alpha \equiv\left\langle\mu F_{P}\right\rangle /\left\langle\mu^{2}\right\rangle^{1 / 2}\left\langle F_{P}^{2}\right\rangle^{1 / 2} .
$$

From $(A 1)$ and $(A 3)$, it can be shown that $\left\langle\mu F_{P}\right\rangle \doteq$ $-\frac{1}{2}\left\langle\mu^{2}\right\rangle$, so that the correlation $\alpha$ is given by

$$
\alpha \doteq-\frac{1}{2}\left\langle\mu^{2}\right\rangle^{1 / 2} /\left\langle F_{P}^{2}\right\rangle^{1 / 2} .
$$

When $\left\langle\mu^{2}\right\rangle$ is small relative to $\left\langle F_{P}^{2}\right\rangle, \alpha$ is clearly small. 
Expressions for $\bar{F}_{P H}$ and $\Delta_{P H}$ in the presence of becomes anomalously scattering heavy atoms

Suppose that all the heavy atoms in the derivative structure have the same ratio of 'real' to 'anomalous' scattering, given by $\kappa=\left(f+f^{\prime}\right) / f^{\prime \prime}$. Then $\mathbf{F}_{\mathbf{H}}$, the nonanomalous part of the heavy-atom structure factor, is perpendicular to $\delta$, the anomalous part of the heavy-atom structure factor:

$$
\delta=\exp (i \pi / 2) F_{\mathbf{H}} / \kappa
$$

so that the total heavy-atom structure factor is given by

$$
\mathbf{F}_{\mathbf{H}}+\boldsymbol{\delta}=\mathbf{F}_{\mathbf{H}}[1+\exp (i \pi / 2) / \kappa] .
$$

If there are several types of anomalously scattering heavy atoms present, $(A 7)$ may be generalized. Let

$$
\mathbf{F}_{\mathbf{H}}=\sum_{j} \mathbf{F}_{\mathbf{H}, j}
$$

and

$$
\boldsymbol{\delta}=\sum_{j} \boldsymbol{\delta}_{j}=\exp (i \pi / 2) \sum_{j} \mathbf{F}_{\mathbf{H}, j} / \kappa_{j}
$$

where $\mathbf{F}_{\mathbf{H}, j}, \boldsymbol{\delta}_{j}$ and $\kappa_{j}$ refer to all atoms of a particular type. Now define the angles $\alpha$ and $\omega$ by

$$
\begin{gathered}
\mathbf{F}_{\mathbf{H}} \equiv\left|\mathbf{F}_{\mathbf{H}}\right| \exp (i \alpha), \\
\boldsymbol{\delta} \equiv|\boldsymbol{\delta}| \exp (i \alpha+i \pi / 2+i \omega)
\end{gathered}
$$

where $\omega$ is zero if all heavy atoms are identical. We may now define an effective value of $\kappa$ which is related to the ratio of 'real' to 'anomalous' scattering for this particular reflection:

$$
\kappa \equiv\left[\left(\left|\mathbf{F}_{\mathbf{H}}\right| /|\boldsymbol{\delta}|\right)-\sin \omega\right] / \cos \omega
$$

where $\kappa$ is equal to each $\kappa_{j}$ if all atoms are identical. Equations $(A 9)-(A 12)$ may now be combined to yield

$\mathbf{F}_{\mathbf{H}}+\boldsymbol{\delta}=\mathbf{F}_{\mathbf{H}}[1+\exp (i \pi / 2) / \kappa] /\left[1-|\boldsymbol{\delta}| \sin (\omega) /\left|\mathbf{F}_{\mathbf{H}}\right|\right]$.

If it is assumed that the ratio $\kappa_{j}$ of real to anomalous scattering is large for all the heavy atoms present, then $|\boldsymbol{\delta}| \sin (\omega) /\left|\mathbf{F}_{\mathbf{H}}\right|$ is nearly always small compared with unity. If this term is ignored, $(A 14)$

$$
\mathbf{F}_{\mathbf{H}}+\boldsymbol{\delta} \doteq \mathbf{F}_{\mathbf{H}}[1+\exp (i \pi / 2) / \kappa] .
$$

Equation $(A 15)$ cannot be used directly to calculate the total heavy-atom structure factor from its nonanomalous part because the factor $\kappa(A 13)$ depends on both $\delta$ and $\mathbf{F}_{\mathbf{H}}$ and may vary from reflection to reflection. In order to avoid this difficulty, we assume that the factor $\kappa$, based on $\boldsymbol{\delta}$ and $\mathbf{F}_{\mathbf{H}}$, is the same as that which can be calculated from the heavy-atom model. Then

$$
\boldsymbol{\delta} \doteq\left(\mathbf{f}_{\mathbf{H}}+\boldsymbol{\eta}\right) \exp (i \pi / 2) / \kappa
$$

where $\kappa$ is calculated from the heavy-atom model using expressions analogous to $(A 11)-(A 13)$.

Now we may obtain expressions for the total derivative structure factor including anomalous-scattering effects. Once again, let $\mathbf{F}_{\mathbf{P H}}$ be the derivative structure factor due to the non-anomalous part of the scattering from the derivative structure:

$$
\mathbf{F}_{\mathbf{P H}} \equiv \mathbf{F}_{\mathbf{P}}+\boldsymbol{\mu}+\mathbf{f}_{\mathbf{H}}+\boldsymbol{\eta} \text {. }
$$

Then the total structure factor for one element of this Friedel pair may be written as (see Fig. 1)

$$
\mathbf{F}_{\mathbf{P H}}^{+}=\mathbf{F}_{\mathbf{P H}}+\boldsymbol{\delta}
$$

and the complex conjugate of the total structure factor for the other element of this Friedel pair may be written as

$$
\mathbf{F}_{\mathbf{P H}}^{-*}=\mathbf{F}_{\mathbf{P H}}-\boldsymbol{\delta} .
$$

With first-order approximations, this leads directly to (18) and (19).

\section{References}

Blow, D. M. \& CRICK, F. H. C. (1959). Acta Cryst. 12, 794-802. Blow, D. M. \& MAtThews, B. W. (1973). Acta Cryst. A29, 56-62. EINSTEIN, R. J. (1977). Acta Cryst. A33, 75-85.

Green, E. A. (1979). Acta Cryst. A35, 351-359.

HAMILTON, W. C. (1964). Statistics in Physical Science. New York: Ronald Press.

Matthews, B. W. (1966). Acta Cryst. 20, 82-86.

NORTH, A. C. T. (1965). Acta Cryst. 18, 212-216.

TEN EYCK, L. F. \& ARNONe, A. (1976). J. Mol. Biol. 100, 3-11.

Terwilliger, T. C. \& EISENBERG, D. (1982). J. Biol. Chem. 257, 6010-6015, 6016-6022.

Wilson, A. J. C. (1949). Acta Cryst. 2, 318-321. 